Urologe $2011 \cdot 50[$ Suppl 1]:7-8

DOI 10.1007/s00120-011-2643-2

Online publiziert: 26. August 2011

๑) Springer-Verlag 2011
P. Anheuser

Klinik für Urologie und Kinderurologie, St.-Antonius-Hospital Eschweiler, Eschweiler

\title{
Auswahlverfahren wissenschaftlicher Beiträge zum DGU-Kongress 2011
}

Die Abstracteinreichung zum 63. Jahrestag der Deutschen Gesellschaft für Urologie (DGU) vom 14.-17.09.2011 in Hamburg erfolgte auch in diesem Jahr vollständig elektronisch. Für einen nahe$\mathrm{zu}$ reibungslosen Ablauf sorgte wie auch im vergangenen Jahr die Fa. Pharmaservice aus Hannover. Vom 15.12.2010 bis zum 31.01.2011 konnten Beiträge über das Internetportal eingereicht werden. Die Zuordnung wurde dabei in 44 bewährte Kategorien vorgenommen.

Die Begutachtung der einzelnen Abstracts erfolgte themenbezogen durch jeweils mindestens 3 Gutachter, die eine Bewertung der Beiträge durchführten und Vorschläge für die Beitragspräsentation in Poster- oder Vortragssitzungen machten. Die abschließende Beurteilung nahmen die 25 Mitglieder der Programmkommission vor, von denen 22 themenspezifisch als Obergutachter tätig waren und die abschließende Bewertung vornahmen. Insgesamt waren 118 Gutachter und an diesem Auswahlverfahren beteiligt.

In diesem Jahr konnten wir erstmalig auch die Weitergabe der eingereichten Abstracts an die Gutachter und deren Bewertung sowie die abschließende Begutachtung durch Obergutachter und Programmkommission elektronisch durchführen, was zu einer deutlichen Zeitersparnis führte.

Zur Annahme kamen alle Beiträge bis zu einer Schwellennote, die in etwa dem Median der statistischen Verteilung entsprach. Abgelehnt wurden Beiträge, deren Daten oder Datensätze bereits in anderer Form unter ähnlicher Fragestellung ange- meldet bzw. berücksichtigt worden sind. Über die Annahme von Beiträgen mit inhomogener Benotung durch die Gutachter entschieden schlussendlich die Obergutachter bzw. die Programmkommission. Dabei wurden Aspekte der Datengewinnung und -menge sowie deren Auswertung berücksichtigt, ebenso Originalität und Aktualität der Thematik.

Insgesamt wurden 758 Abstracts eingereicht, die Anzahl variierte bei den einzelnen Themen deutlich (o-49) und be- trug im Mittel 17. Von diesen eingereichten Beiträgen wurden 468 angenommen, was einer Quote von 61,7\% entspricht. Dabei wurden 181 Abstracts einer Posterpräsentation, 277 Beiträge einer Vortragssitzung und 10 Beiträge einer Filmsitzung zugeordnet. Nach der endgültigen Einteilung wurden 16 Abstracts von den Autoren zurückgezogen. Eine Übersicht der einzelnen Beiträge finden Sie themenbezogen in der unten angeführten $\bullet$ Tab. 1.

\section{Tab. 1 Anzahl der eingereichten und angenommenen Abstracts}

\begin{tabular}{|c|c|c|c|c|c|}
\hline Nr. & Thema & $\begin{array}{l}\text { Einge- } \\
\text { reicht }\end{array}$ & $\begin{array}{l}\text { Ange- } \\
\text { nommen }\end{array}$ & $\begin{array}{l}\text { Abge- } \\
\text { lehnt }\end{array}$ & $\begin{array}{l}\text { Zurück- } \\
\text { gezogen }\end{array}$ \\
\hline 1 & BPH & & & & \\
\hline 1.1 & Experimentell & 7 & 6 & 1 & 0 \\
\hline 1.2 & Klinisch & 22 & 10 & 12 & 0 \\
\hline 2 & Unterer Harntrakt, Urodynamik, Neurourologie & & & & \\
\hline 2.1 & Experimentell & 18 & 9 & 9 & 0 \\
\hline 2.2 & Diagnostik & 9 & 7 & 2 & 0 \\
\hline 2.3 & Therapie & 12 & 9 & 3 & 0 \\
\hline 3 & Niere, oberer Harntrakt & & & & \\
\hline 3.1 & Physiologie, Pathophysiologie & 1 & 1 & 0 & 0 \\
\hline 3.2 & Transplantation & 8 & 7 & 1 & 0 \\
\hline 4 & Nieren- und Nebennierentumoren & & & & \\
\hline 4.1 & Experimentell & 23 & 19 & 3 & 1 \\
\hline 4.2 & Diagnostik & 10 & 9 & 1 & 0 \\
\hline 4.3 & Therapie & 32 & 18 & 14 & 0 \\
\hline 5 & Urothelkarzinom & & & & \\
\hline 5.1 & Experimentell & 31 & 19 & 10 & 2 \\
\hline 5.2 & Diagnostik & 22 & 22 & 0 & 0 \\
\hline 5.3 & Therapie oberflächlicher Tumoren & 4 & 4 & 0 & 0 \\
\hline 5.4 & Therapie fortgeschrittener Tumoren & 32 & 19 & 13 & 0 \\
\hline 6 & Prostatakarzinom & & & & \\
\hline 6.1 & Experimentell & 49 & 19 & 29 & 1 \\
\hline 6.2 & Diagnostik & 39 & 19 & 20 & 0 \\
\hline
\end{tabular}




\section{Tab. 1 Anzahl der eingereichten und angenommenen Abstracts}

Nr. Thema

6.3 Therapie lokalisierter Tumoren

6.4 Therapie fortgeschrittener Tumoren

7 Tumoren des äußeren Genitale

7.1 Hodentumoren

7.2 Peniskarzinome

8 Inkontinenz und Beckenboden

8.1 Inkontinenz bei Mann und Frau

8.2 Beckenbodenrekonstruktion

9 Andrologie

9.1 Sexuelle Dysfunktion

9.2 Hypogonadismus

9.3 Infertilität

10 Kinderurologie

11 Uro-Traumatologie

12 Infektionen/Entzündungen

12.1 Obere Harnwege

12.2 Urogenitaltrakt

13 Urolithiasis

13.1 Experimentell

13.2 Diagnostik

13.3 Therapie

14 Uro-Imaging

15 Operationstechniken

15.1 Rekonstruktive Eingriffe

15.2 Harnableitung

15.3 Endoskopische Techniken

15.4 Laparoskopie

15.5 Roboterchirurgie

16 Palliativmedizin und Schmerztherapie

17 Prävention, Umwelt- und Komplementärmedizin

18 Soziologie und Psychosomatik

19 Krankenhausökonomie/DRG/Qualitätssicherung

20 Falldemonstrationen/interessante Fälle („Albträume“)

21 Geschichte der Urologie

Gesamt
Einge- Ange- Abge- Zurückreicht nommen lehnt gezogen

$\begin{array}{llll}77 & 48 & 26 & 3\end{array}$

14600

126

99

$\begin{array}{ll}5 & 1 \\ 0 & 0\end{array}$

$27 \quad 16$

110

95

$\begin{array}{llll}8 & 6 & 2 & 0\end{array}$

$\begin{array}{llll}5 & 3 & 1 & 1\end{array}$

$\begin{array}{llll}7 & 5 & 2 & 0\end{array}$

$22 \quad 18 \quad 3 \quad 1$

$0 \quad 0 \quad 0 \quad 0$

$0 \quad 0$

159

$\begin{array}{llll}10 & 8 & 2 & 0\end{array}$

$\begin{array}{llll}9 & 6 & 3 & 0\end{array}$

$23 \quad 18 \quad 5 \quad 0$

$\begin{array}{llll}11 & 5 & 4 & 2\end{array}$

$\begin{array}{llll}28 & 14 & 12 & 2\end{array}$

$\begin{array}{llll}7 & 4 & 3 & 0\end{array}$

$\begin{array}{llll}10 & 5 & 5 & 0\end{array}$

$\begin{array}{llll}31 & 18 & 12 & 1\end{array}$

$\begin{array}{llll}37 & 18 & 19 & 0\end{array}$

$\begin{array}{llll}4 & 1 & 3 & 0\end{array}$

1

\section{8}

18

26

11

758
Auch in diesem Jahr wurden die Videobeiträge von der Filmkommission getrennt von den übrigen Abstracts bewertet. Es wurden alle 10 Anmeldungen berücksichtigt.

Ein herzliches Dankeschön gilt an dieser Stelle allen beteiligten Gutachtern für Ihre exzellente, kritische und zeitnahe Begutachtung der Beiträge sowie Obergutachtern und der Programmkommission für die kompetente und kollegiale Zusammenarbeit. Danken möchten wir auch der Firma Pharmaservice für die konstruktive Zusammenarbeit.

Wir freuen uns auf einen interessanten 63. DGU-Kongress mit Ihnen in Hamburg.

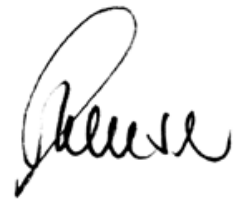

\section{P. Anheuser}

\section{Korrespondenzadresse}

Dr. P. Anheuser

Klinik für Urologie und Kinderurologie, St.-Antonius-Hospital Eschweiler, Dechant-Deckers-Straße 8, 52249 Eschweiler petra.anheuser@sah-eschweiler.de 